\title{
FORMULATION AND EVALUATION OF SOLID DISPERSIONS OF NABUMETONE
}

\author{
*Hyma Ponnaganti ${ }^{1}$, Renuka $\mathbf{R}^{1}$, Rajani C. ${ }^{1}$, S.D. Shanmuga kumar ${ }^{2}$ \\ ${ }^{1}$ Associate Professor, Pharmaceutics Department, Jyothishmathi College of Pharmacy, Hyderabad, India \\ ${ }^{2}$ Principal, Jyothishmathi College of Pharmacy, Hyderabad, India \\ *Corresponding Author's Email: rk_hyma@yahoo.com
}

\begin{abstract}
Solid dispersions are an effective method of increasing the solubility and bioavailability of poorly soluble drugs. In the present study it is aimed at to enhance solubility of Nabumetone a poorly soluble anti-inflammatory drug by solid dispersion technique by employing solvent evaporation technique using PEG 4000 And PEG 6000 as carriers. The formulations were prepared in ratios of $1: 1,1: 2,1: 3,1: 4$ and characterized by FTIR, XRD, and dissolution studies. The prepared solid dispersions showed excellent improvement of drug solubility due to mean drug particle size reduction which lead to increase drug dissolution and bioavailability.
\end{abstract}

Key words: Nabumetone, PEG4000, PEG6000, FTIR, XRD.

\section{INTRODUCTION:}

Nabumetone is a non-steroidal anti-inflammatory drug used to treat pain or inflammation caused by arthritis. It has poor oral absorption due to poor solubility and low dissolution rate. ${ }^{1}$ Therefore a strategy was developed pharmaceutically to increase its solubility and dissolution by the method of solid dispersions. Solid dispersions is the most popularly employed techniques which could improve dissolution rate and solubility of poorly water soluble drugs by reducing particle size, fractionating to amorphous form and improving the wettability. ${ }^{2}$ This transformation to amorphous to amorphous form of drug from crystalline state attributes to increase in solubility and improves bioavailability. ${ }^{3}$

As reported even hydrogen bonding formed between drug and carriers is another reason for improved solubility and decreased crystallinity of drug in solid dispersions. ${ }^{4,5}$ The formulations were prepared by solvent evaporation technique and later subjected to various evaluation tests like FTIR,XRD and dissolution studies and compared to pure drug. Comparison of results with those for pure drug powder and physical mixtures of the drug and carrier can help to indicate the mechanism by which the carrier improves dissolution: via solubilization and wetting effects which could be affected by a simple mixture of the components, or by formation of a solid dispersion/solution. ${ }^{6,7} \mathrm{~A}$ well-designed release experiment will show whether the solubility of the drug and its dissolution rate has been enhanced, and also whether the resulting supersaturated solution is stable or tends to precipitate quickly.

\section{MATERIALS AND METHODS}

\section{Solvent evaporation technique: ${ }^{11,12}$}

Solid dispersions were prepared by solvent evaporation method. The carrier PEG 4000 and PEG 6000 are used by adding amounts of nabumetone corresponding the ratio 1:1;1:2,1:3 and 1:4 was accurately weighed and mixed properly. This physical mixture was solubilised in common solvent that is in methanol $(5 \mathrm{ml})$. The solvent was allowed to evaporate in hot air oven at $45 \mathrm{C} \pm 10 \mathrm{c}$. The process of evaporation was opted until the constant weight was obtained. The formulation was kept in desiccators for 24hrs under vacuum. Then, solid dispersion formulation was pulverized using a porcelain mortar and pestle. The pulverized powder was classified using the sieves (size $60 \#)$ and the powder was used for study.

\section{Evaluation of prepared solid dispersions:}

\section{Drug content}

The dispersion system equivalent to $10 \mathrm{mg}$ of Nabumetone Phosphate Buffer was taken in $100 \mathrm{ml}$ vol. Flask and dissolved in methanol. The volume was made up to mark with buffer and filtered. One $\mathrm{ml}$ of filtrate was further diluted to $10 \mathrm{ml}$ with methanol and absorbance was recorded at $271 \mathrm{~nm}$. The amount of drug in each dispersion system was determined spectrophotometrically. The results were shown in table 2

\section{In Vitro drug release}

In Vitro drug release rate of Nabumetone Phosphate Buffer solid dispersion of different samples was determined using USP dissolution test apparatus. The dissolution medium consisted of 6.8 Phosphate Buffer. Samples of drug, solid dispersion equivalent to $100 \mathrm{mg}$ of drug was spread onto the surface of $900 \mathrm{ml}$ of preheated dissolution medium at 37 C. Aliquots of $5 \mathrm{ml}$ were withdrawn at regular intervals of time $(5,10,15,20$, upto $120 \mathrm{~min})$ and the same is replaced with fresh dissolution medium each time. The samples were measured for absorbance at $271 \mathrm{~nm}$. The graph is plotted by taking percentage drug release on $\mathrm{Y}$-axis and time on $\mathrm{X}$-axis ${ }^{16}$. The results are shown in table $3 \& 4$, graphs in fig $8 \& 9$.

\section{Fourier transforms Infrared spectroscopy}

\section{FT-IR spectra: ${ }^{13}$}

FT-IR spectra were recorded using an FT-IR spectrophotometer (shimadzu).The samples (nabumetone PHOSPHATE BUFFER, PEG6000, and solid dispersions) were previously ground and mixed thoroughly with potassium bromide .Forty scans were obtained at a 
resolution of $4 / \mathrm{cm}$ from $4500 / \mathrm{cm}$. The corresponding graphs are shown in fig $1,2,3 \& 4$.

\section{X-ray diffraction: ${ }^{14,15}$}

The crystalline state of different samples was evaluated with X-ray powder diffraction. Diffraction patterns were obtained using an XPERT-PRO diffract meter (PAN atypical) with a radius of $240 \mathrm{~nm}$. The $\mathrm{Cu} \mathrm{K \alpha}$ radiation ( $\mathrm{K}$ $\alpha 1.54060 \mathrm{~A}^{\circ}$ ) was $\mathrm{Ni}$ filtered. A system of diverging and receiving slits of $1^{\circ}$ and $0.1 \mathrm{~mm}$ respectively was used. The pattern was collected with $40 \mathrm{kv}$ of tube voltage and $30 \mathrm{~mA}$ of tube current and scanned over the $2_{\varphi}$ range of $5-60^{\circ}$. The corresponding results were shown in fig $5,6 \& 7$.

\section{RESULTS:}

Table 1: Formulation of solid dispersions in various ratios:

\begin{tabular}{|l|l|l|l|l|l|l|l|l|}
\hline Contents & F1 & F2 & F3 & F4 & F5 & F6 & F7 & F8 \\
\hline Nabumetone & 1 & 1 & 1 & 1 & 1 & 1 & 1 & 1 \\
\hline PEG 4000 & 1 & 2 & 3 & 4 & - & - & - & - \\
\hline PEG 6000 & - & - & - & - & 1 & 2 & 3 & 4 \\
\hline
\end{tabular}

Table 2: Drug content

\begin{tabular}{|ll|}
\hline Formulation & Drug content (\%) \\
F1 & 9869 \\
F2 & 89.78 \\
F4 & 88.53 \\
F5 & 99.67 \\
F6 & 95.56 \\
F7 & 92.67 \\
F8 & 96.34 \\
\hline
\end{tabular}

Table 3: Dissolution studies of formulations with PEG 4000.

\begin{tabular}{|l|l|l|l|l|l|}
\hline \multicolumn{1}{|c|}{ Time } & \multicolumn{1}{|c|}{ F1 } & F2 & F3 & F4 & Pure Drug \\
\hline & 0 & 0 & 0 & 0 & 0 \\
\hline 10 & 12.34 & 14.4 & 32 & 44 & 9.12 \\
\hline 20 & 20.12 & 34.2 & 34 & 56 & 15.02 \\
\hline 30 & 29.34 & 39.6 & 48 & 67 & 21.21 \\
\hline 40 & 32.34 & 44.3 & 55 & 79 & 25.05 \\
\hline 50 & 40.12 & 51.2 & 62 & 95 & 32.23 \\
\hline 60 & 52.23 & 63 & 70 & 98 & 39.01 \\
\hline
\end{tabular}

Table 4: Dissolution studies of formulations with PEG6000.

\begin{tabular}{|c|c|c|c|c|c|}
\hline Time & F1 & F2 & F3 & F4 & Pure Drug \\
\hline 0 & 0 & 0 & 0 & 0 & 0 \\
\hline 10 & 10.56 & 12.23 & 15.56 & 20.12 & 9.12 \\
\hline 20 & 15.12 & 20.34 & 24.34 & 30.23 & 15.02 \\
\hline 30 & 26.23 & 33.23 & 35.56 & 41.23 & 21.21 \\
\hline 40 & 30.34 & 44.56 & 46.34 & 52.34 & 25.05 \\
\hline 50 & 35.54 & 49.23 & 51.23 & 60.43 & 32.23 \\
\hline 60 & 40.12 & 51.43 & 60.23 & 74.45 & 30.01 \\
\hline
\end{tabular}


Figure 1: FTIR spectra of pure drug

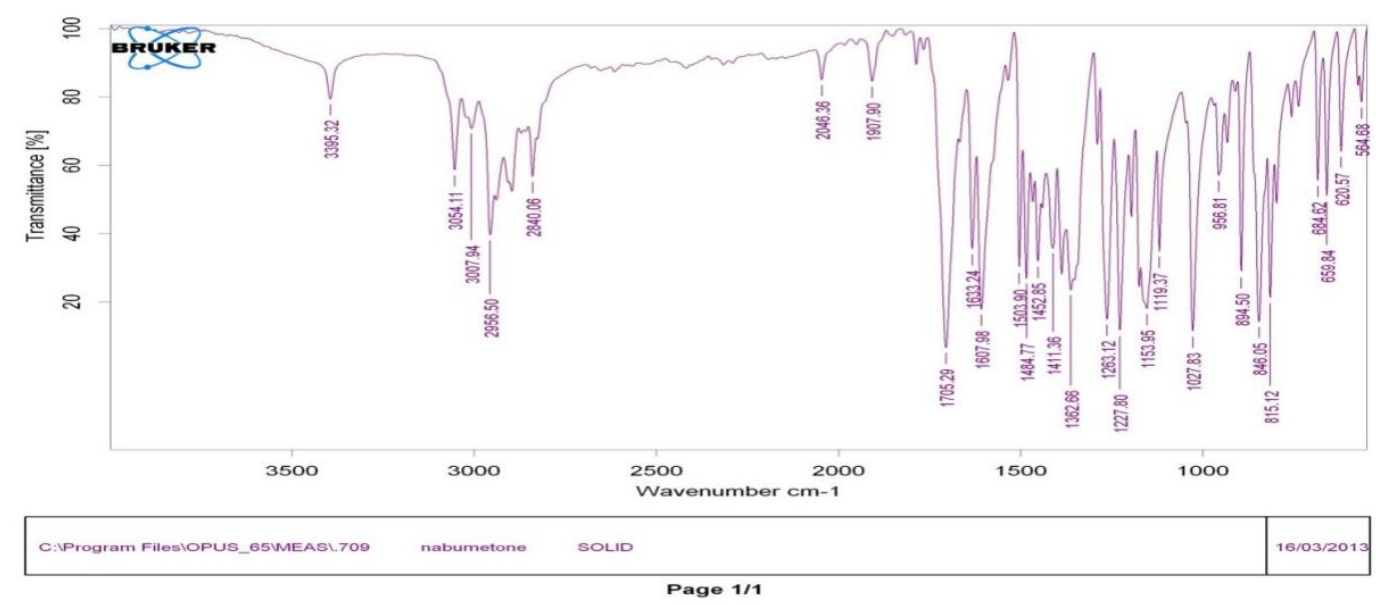

Figure 2: FTIR SPECTRA of PEG 4000

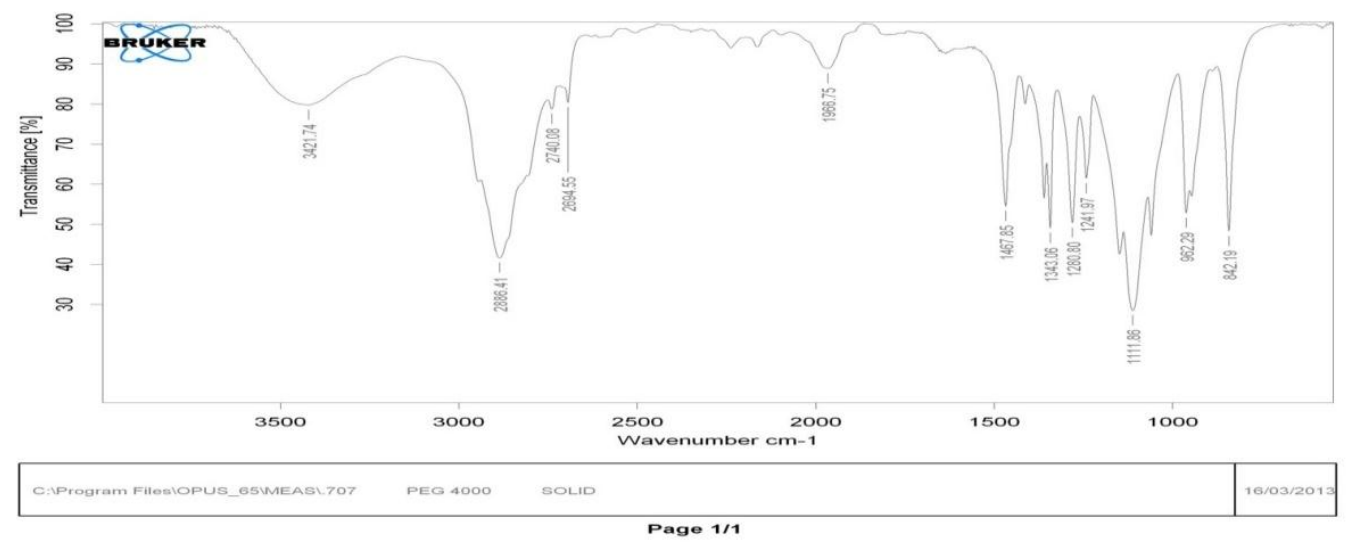

Figure 3: FTIR SPECTRA OF PEG 6000

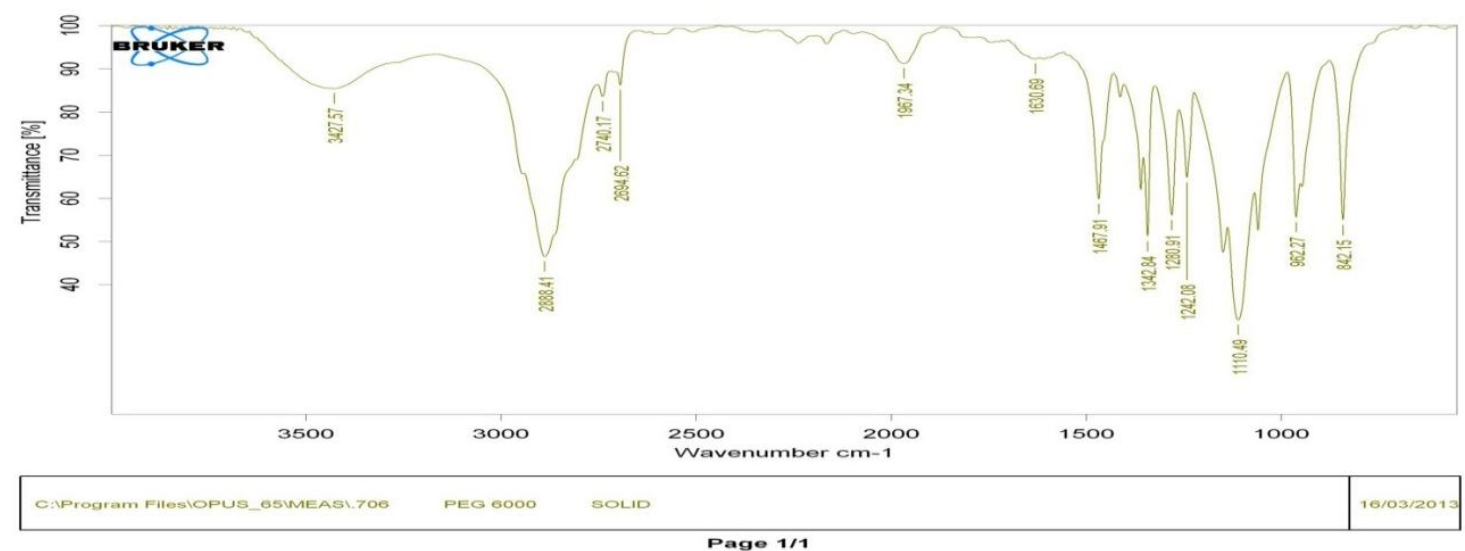

Figure 4: FTIR Spectra of solid dispersion

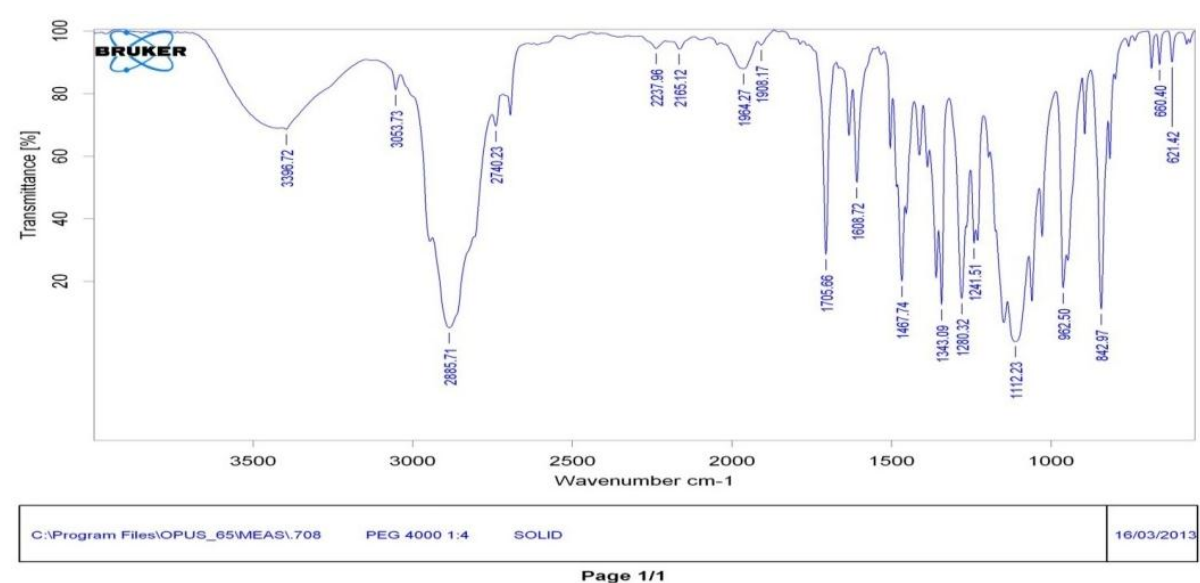


Figure 5: XRD spectra of nabumetone:

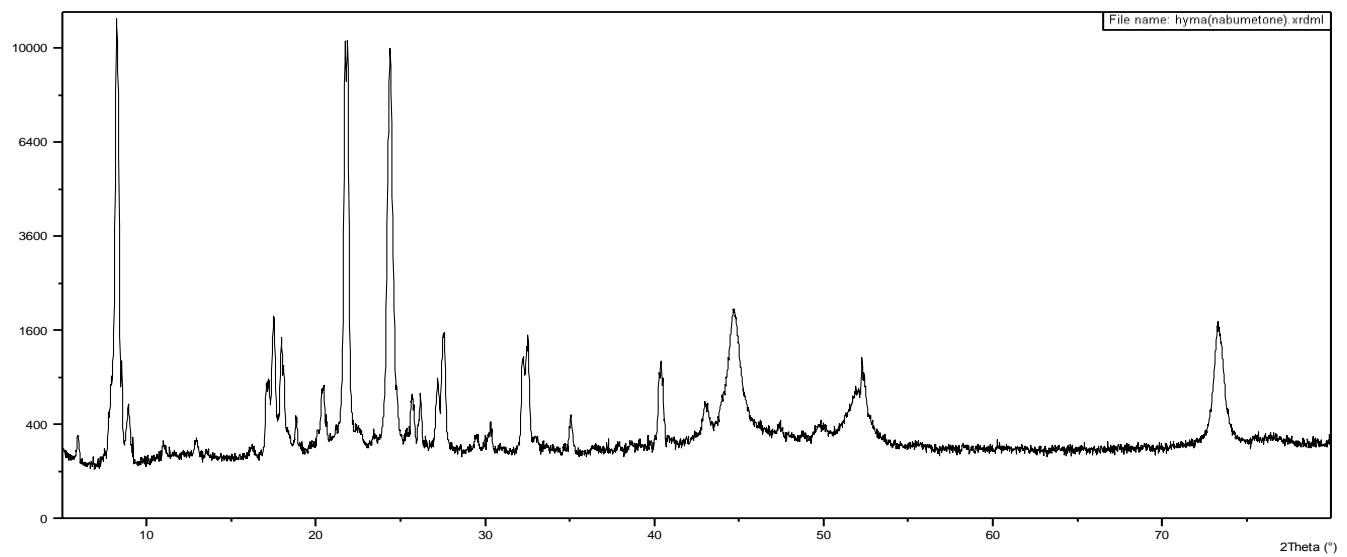

Figure 6: XRD spectra of PEG 4000

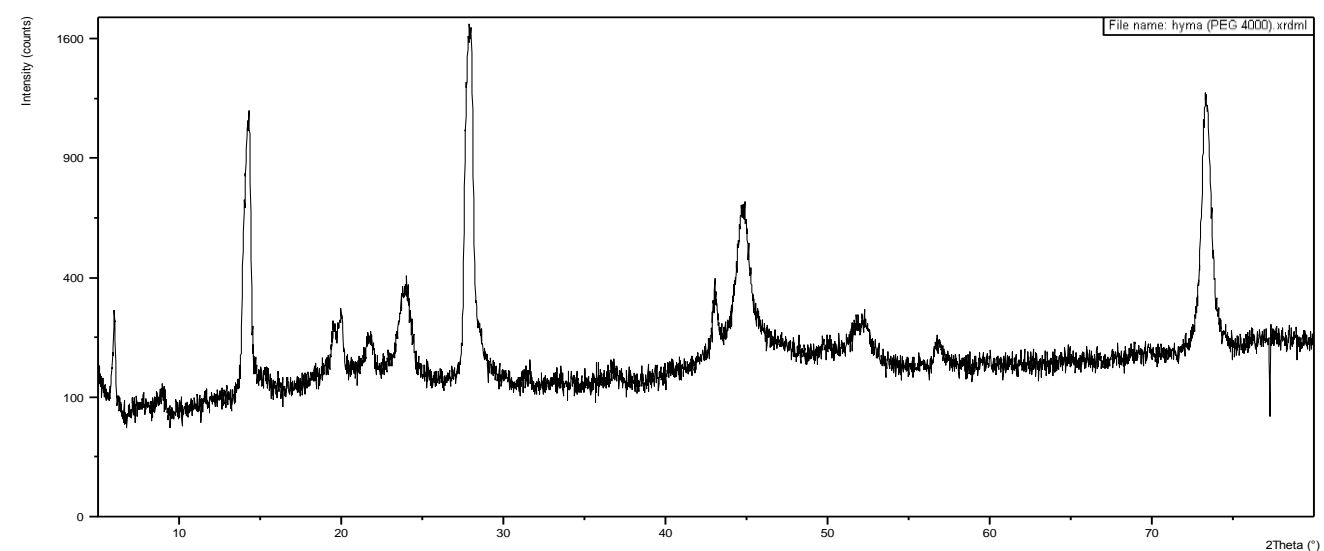

Figure 7: XRD spectra of solid dispersion

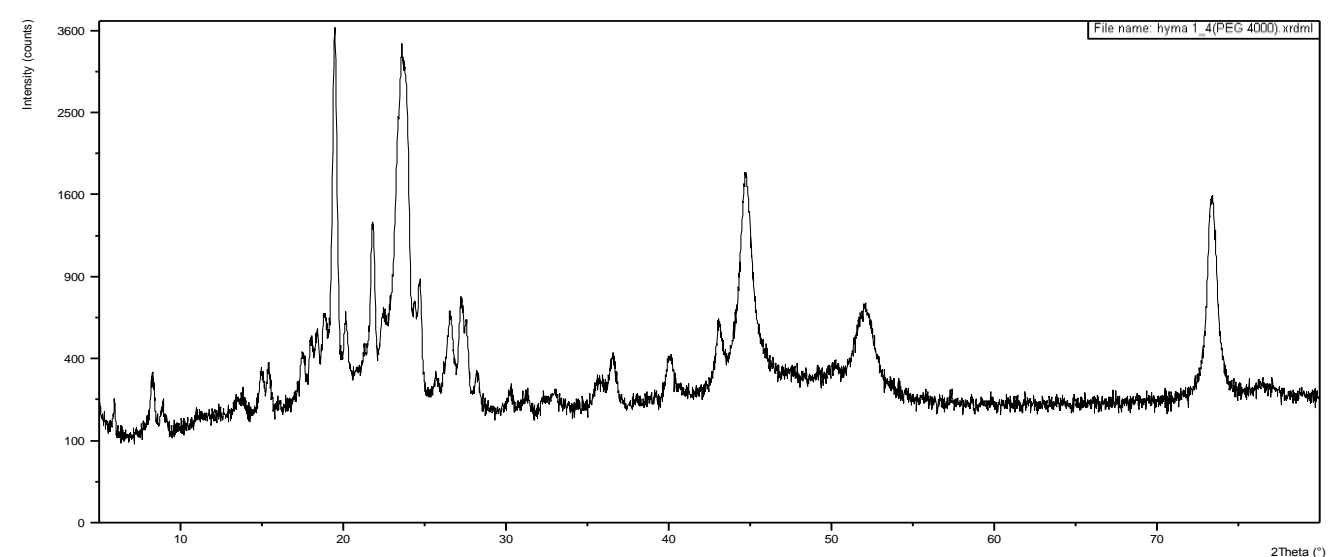

Figure 8: Dissolution profile of solid dispersions with PEG 4000

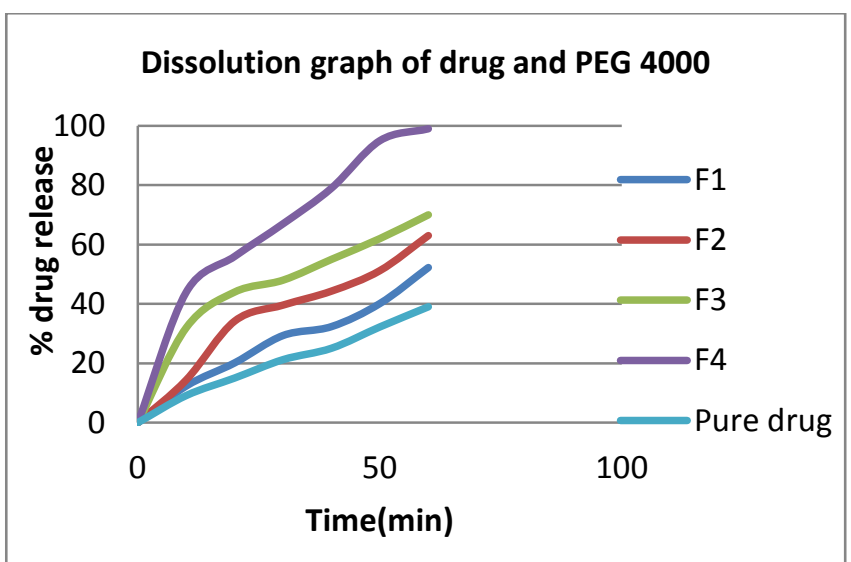

Figure 9: Dissolution profile of solid dispersions with PEG 6000

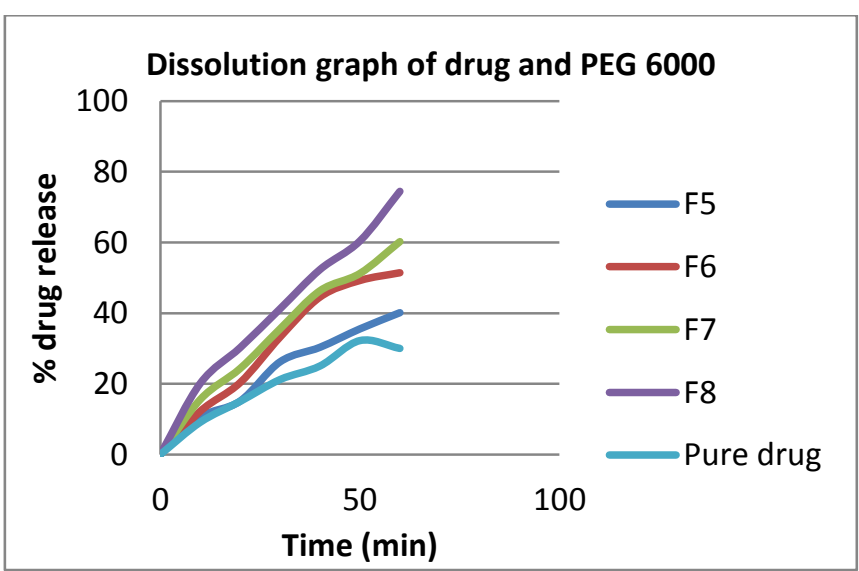




\section{DISCUSSION:}

\section{FTIR spectra:}

As can be seen, a strong absorption band of $\mathrm{c}=0$ stretching at 1705 and $\mathrm{C}=\mathrm{C}$ ring stretching at $1607 ; 1633 ; 1484 ; 150 \mathrm{~cm}-1$. Occurs int the IR spectrum of Nabumetone. The same absorption bands at $1705 \& 1605 \mathrm{~cm}-1$ are present in the spectra of SOLID DISPERSION of Nabumetone and PEG 4000.The absence of any shift of carbonyl stretching band and alkenes ring stretching band suggested that know chemical interaction occurs between Nabumetone and PEG 4000 ${ }^{14}$.

\section{Dissolution Studies}

Invitro Drug Release:-Dissolution profiles of pure drug and solid dispersions show that improved dissolution rate is proportional with carrier and drug ratio. The influence of PEG 6000 \& PEG 4000 on the dissolution of can be explained by the formation of region of high concentration of dissolved polymer at the surface of drug crystals in which the drug can solublise and subsequently diffuse and dilute in the bulk of the solution. At the end of I hour, F1 is $90, \mathrm{~F} 2$ is $92, \mathrm{~F} 3$ is 95 , F4 is 101.12 , F5 is 100.09 , F6 is 99.14 , F7 is 99 and F8 is 98.Based on dissolution studies the optimized formula was found to be which released $99 \%$ at the end of 1 hour

\section{REFERENCES:}

1. Christian Leuner,Jennifer Dressman, Improving drug solubility for oral delivery using solid dispersions, European journal of pharmaceutics, 2000,50,47-60.

2. Verheyen S, Blaton N, Kinget R, Van den Mooter G, Mechanism of increased dissolution of diazepam and temazepam from polyethylene glycol 6000 solid dispersions, International journal of pharmaceutics, 2002,249,45-58

3. Mercedes Fernandez,Ines Carmen Rodriguez,Maria Victoria Margarit and Antoni Cerezo, Characterization of solid dispersions of piroxicam/polyethylene glycol 4000, International journal of pharmaceutics, 84(1992)197-202.

4. Lioyd GR, Craig DQM, Smith A, A calorimetric investigation into the interaction between paracetamol and polyethylene glycol 4000 in physical mixes and solid dispersions, European International journal of pharmaceutics, 1999, 48, 59-65.

5. Wu JX.; Yang M. van den Berg F, Pajander J, Rades T, Rantanen J. Influence of solvent evaporation rate and formulation factors on solid dispersion physical stability. Eur. J. Pharm. Sci. 2011, 44, 610-620.

6. Al-Obaidi H.; Brocchini S; Buckton G. Anomalous properties of spray dried solid dispersions. J. Pharm Sci. 2009, 98, 4724-4737.

7. Kojima T; Higashi K; Suzuki T; Tomono K; Moribe K; Yamamoto K. Stabilization of asupersaturated solution of mefenamic acid from a solid dispersion with EUDRAGIT® EPO. Pharm. Res. 2012, 29, 2777-2791. compared to pure drug releasing 39.01 at the end of one hour. Therefore $\mathrm{f} 4$ was further investigated for further evaluation ${ }^{17}$.

\section{XRD studies:}

1n XRD study, characteristic peak of drug appeared in 20 range of $10^{\circ}-30^{\circ}$ indicating that the unprocessed drug was a crystalline material. The pure drug exhibit, its characteristic diffraction peaks at various diffraction angles indicating the presence of crystallinity. The X-Ray diffraction study of solid dispersion showed broadening and reduction of major drug peaks indicating that mostly an amorphous form existed in solid dispersion. These results could explain the observed enhancement of solubility and rapid dissolution of drug in solid dispersion.

\section{ACKNOWLEDGEMENTS:}

Authors are deeply thankful to the management Jyothishmathi college of pharmacy, director Prof.M.Sainarayana, for their constant support and kind help in carrying out the work, a sincere thanks to Mr.Vishveshwar rao and Mr.Srinivas Rao for their support and assistance, heartfelt thanks to Lalitha college of pharmacy,Hyderabad for FTIR analysis and Central University ,Hyderabad for XRD studies.

8. Greco S; Authelin JR; Leveder C; Segalini A. A practical method to predict physical stability of amorphous solid dispersions. Pharm. Res. 2012, 29, 2792-2805.

9. Willart JF; Descamps M. Solid state amorphization of pharmaceuticals. Mol. Pharm. 2008, 5,905-920.

10. Brahmankar DM, Jaiswal SB, Biopharmaceutics \& Pharmaceutics, First Edition, 1995, 335.

11. Potluri RH; Bandari S; Jukanti R.; Veerareddy PR. Solubility enhancement and physicochemical characterization of carvedilol solid dispersion with Gelucire 50/13. Arch. Pharm.Res. 2011, 34, 51-57.

12. El-Fattah A, Salib NN, El-Assik M. A new approach for controlling the release rate of pheniramine aminosalicylate via solid dispersions. Drug Develop Ind Pharm 1984,10, 649-66.

13. Hasegawa A, Nakagawa H, Sugimoto L. Bioavailability and stability of nifedipine-enteric coating agent solid dispersion. Chem Pharm Bull 1985,33, 388-91.

14. Pignatello R, Ferro M, De Guidi G, Salemi G, Vandelli MA, Guccione $\mathrm{S}$, et al. Preparation, characterization and photosensitivity studies of solid dispersions of diflunisal and Eudragit RS 100 and RL 100. Int J Pharm 2001,218,27-42.

15. Ammar HO, Khalil RM. Preparation and evaluation of sustained release solid dispersions of drugs with eudragit polymers. Drug Develop Ind Pharm , 1997, 23, 1043-54. 